\title{
Central Decomposition of Invariant States Applications to the Groups of Time Translations and of Euclidean Transformations in Algebraic Field Theory
}

\author{
D. Kastler and M. MebKhout
}

UER Pluridisciplinaire de Marseille-Luminy. Université d'Aix-Marseille II et Centre de Physique Théorique, CNRS, Marseille, France

\section{G. LOUPIAS}

Physique Mathematique

Equipe de Recherche Associee au CNRS, Département de Mathématiques, Université des Sciences et Technique du Languedoc, Montpellier, France

\section{MICHEL}

Institut des Hautes Etudes Scientifique, 91440 Bures-Sur-Yvette. France

\author{
Received March 15, 1972
}

\begin{abstract}
With $\mathfrak{A}$ a $C^{*}$-algebra with unit and $g \in G \rightarrow \alpha_{g}$ a homomorphic map of a group $G$ into the automorphism group of $G$, the central measure $\mu_{\Phi}$ of a state $\Phi$ of $\mathfrak{A}$ is invariant under the action of $G$ (in the state space of $\mathfrak{A}$ ) iff $\Phi$ is $\alpha$-invariant. Furthermore if the pair $\{\mathfrak{A}, G\}$ is asymptotically abelian, $\Phi$ is ergodic iff $\mu_{\Phi}$ is ergodic. Transitive ergodic states (corresponding to transitive central measures) are centrally decomposed into primary states whose isotropy groups form a conjugacy class of subgroups. If $G$ is locally compact and acts continuously on $\mathfrak{A}$, the associated covariant representations of $\{\mathfrak{U}, \alpha\}$ are those induced by such subgroups. Transitive states under time-translations must be primary if required to be stable. The last section offers a complete classification of the isotropy groups of the primary states occurring in the central decomposition of euclidean transitive ergodic invariant states.
\end{abstract}

\section{Introduction}

The general setting of this paper is the one encountered in algebraic field theory: we are given a $C^{*}$-algebra $\mathfrak{U}$-(in physics the "quasi-local algebra", norm completion of the set of local obervables) and a locally compact group $G$ acting as automorphisms of $\mathfrak{A}$ (one of the invariance groups of the physical theory). We recall that the states of $\mathfrak{U}$ (normalized positive functionals) are interpreted as the states of the physical system. The states of $\mathfrak{U}$ invariant under $G$ are of particular interest both mathematically and physically. Mathematically they provide a "non commutative generalization" of the invariant measures basic in ergodic 
theory; the representations of $\mathfrak{A}$ which they generate are "covariant" in the sense that the $G$-automorphisms are implemented by a unitary representation of $G$ in the representation Hilbert space. The non commutative analogues of the ergodic invariant measures of classical ergodic theory are the extremal invariant states, the so called ergodic states. Physically the interest of invariant states resides in the description of thermodynamical equilibrium states of infinite quantum systems, whereby $G$ is either the time evolution group or some other invariance group of the physical theory, e.g. the euclidean or the gauge group. The most basic role in connection with equilibrium states is played by the time translation group: physical equilibrium states are by definition time-invariant and we expect them to be ergodic if they represent pure thermodynamical phases. On the other hand invariant ergodic states with respect to other symmetry groups (e.g. the euclidean or the gauge group) seem of interest for physics because a wide class of them is shown to be integrals of states with lower symmetry, i.e. invariant only under a subgroup of $G[1,2,3]$. Such states "with broken symmetry" frequently occur in nature as equilibrium states of physical systems, e.g. ferromagnets, crystals, superfluids, superconductors.

In this paper we study with the above motivation the central decomposition of invariant, and especially of ergodic, states. Given an arbitrary state $\Phi$ of the $C^{*}$-algebra $\mathfrak{A}$ one can define a unique "central decomposition of $\Phi$ " into primary ${ }^{1}$ states

$$
\Phi=\int \psi d \mu_{\Phi}(\psi) .
$$

This decomposition corresponds to the unique central reduction of the representation of $\mathfrak{A}$ generated by $\Phi$ and assigns uniquely to each state $\Phi$ of $\mathfrak{Y}$ its "central measure" $\mu_{\Phi}$ which can be characterized by properties of (*) making no reference to the central reduction of representations $[5,6]$. After a first section recalling the main properties of the central decomposition as well as some known equivalences between definitions of continuity for the action of $G$ on $\mathfrak{A}$, we describe in Section 2 the basic features of central decompositions of invariant states: we show that $\Phi$ is $G$-invariant if and only if $\mu_{\Phi}$ is invariant under the transposed action

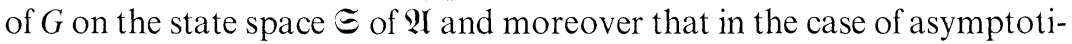
cally abelian systems, $\Phi$ is ergodic if and only if $\mu_{\Phi}$ is ergodic (in fact the restriction to the asymptotically abelian case can be avoided if we define ergodicity of states as the feature that $G$ acts ergodically on the center of the associated representation rather than as e.g. convex extremality). The latter result provides an immediate classification of ergodic states

${ }^{1}$ A state is called primary whenever the weak closure of the set of representatives in the representation it generates is a von Neumann factor. 
into transitive ergodic states (having transitive ergodic measures) and the ones which are not. The first, more elementary, type is considered in Section 3 where one shows that the central decomposition $(*)$ can then be effected by integrating over a homogeneous space of $G$, namely the orbit of $G$ (in the state space $\Xi$ ) on which $\mu_{\Phi}$ is concentrated. We furthermore show that the covariant representation of $\mathfrak{Q}$ associated to $\Phi$ is an induced representation, whereby the inducing subgroup is the stabilizer (isotropy subgroup) of the points of that orbit.

The last two sections provide physical applications. Section 4, devoted to the group of time-translations, offers a discussion of the connection between primariness of an ergodic transitive invariant state and a "stability" property. Section 5 contains a classification of all homogeneous spaces of the euclidean group which carry a bounded invariant measure. One finds, of course, amongst them, those corresponding to the "crystallographic groups", but also other cases some of which at least occur in nature.

A first version of the initial sections of this paper was distributed a few years ago as a preprint under the co-authorship of Rudolf Haag, who decided to our regrets to withdraw from the present final version. In fact the whole project initiated in an attempt to answer the following question raised by Prof. Haag: why is it that in all physically meaningful known models equilibrium stages turn out to be primary? We would like to express our hearty thanks to Prof. Haag for the incentive the provided to this paper and we hope to give a satisfying answer to this question in our Section 4.

\section{§1. Generalities}

Let $\mathfrak{A}$ be a countably generated $C^{*}$-algebra with unit $I, \mathfrak{A}^{*}$ its topological dual space and $\subseteq$ the state space of $\mathfrak{A}$ (i.e. the set of elements $\Phi \in \mathfrak{I}^{*}$ such that $\Phi\left(A^{*} A\right) \geqq 0$ for all $A \in \mathfrak{A}$ and $\left.\Phi(I)=\|\Phi\|=1\right)$. Under the $w^{*}$-topology inherited from $\mathfrak{I}^{*}$ (with neighbourhoods of zero the $U_{\varepsilon,\left\{A_{i}\right\}}=\left\{\Phi \in \mathbb{S}|| \Phi\left(A_{i}\right) \mid<\varepsilon, i=1,2, \ldots n\right\}, \varepsilon>0, A_{1}, A_{2}, \ldots A_{n} \in \mathfrak{A}, n$ arbitrary integer), $\subseteq$ is compact with a countable basis of open sets and thus metrisable. We denote by $\mathfrak{I}^{* *}$ the bidual (enveloping Von Neuman algebra $[7 ; \S 12])$ of $\mathfrak{A}$ and by 3 the center of $\mathfrak{A}^{* *}$.

For a $\Phi \in \mathfrak{S}, \pi_{\Phi}$ denotes the representation of $\mathfrak{A}$ obtained from $\Phi$ by means of the Gelfand-Segal construction $[8,9]$ (acting on the Hilbert space $\mathscr{H}_{\Phi}$ with cyclic vector $\Omega_{\Phi}$ such that $\left.\Phi(A)=\left(\Omega_{\Phi}\left|\pi_{\Phi}(A)\right| \Omega_{\Phi}\right), A \in \mathfrak{U}\right)$. $\Phi$ and $\pi_{\Phi}$ have unique $\sigma$-continuous extensions to $\mathfrak{I}^{* *}[7, \S 12]$ for which we use the same symbols $\Phi, \pi_{\Phi}$. Furthermore we denote by $\mu_{\Phi}$ and $\Lambda_{\Phi}$ the respective central (Radon) measure of $\Phi$ and corresponding central homomorphism with the following defining properties: $\mu_{\Phi}$ is a regular measure on the compact set $\Theta$ such that one has, for each $A \in \mathfrak{A}$ 
and $Z \in 3$,

$$
\begin{gathered}
\Phi(Z A)=\int_{\Xi} \Lambda_{\Phi}(Z)(\psi) \cdot \psi(A) d \mu_{\Phi}(\psi) \\
\left(=\left\langle\mu_{\Phi}, \Lambda_{\Phi}(Z) \cdot \hat{A}\right\rangle \text { with } \hat{A}(\psi)=\psi(A), \psi \in \Xi\right)
\end{gathered}
$$

where $\Lambda_{\Phi}: Z \rightarrow \Lambda_{\Phi}(Z)$ is a ( $\sigma$-continuous) $*$-homomorphism of 3 onto the Von Neumann algebra $L^{\infty}\left(\Im, \mu_{\Phi}\right)$ of essentially bounded $\mu$-measurable functions on $\Xi$. As shown by Sakai in [5] this definition ensures the existence and uniqueness of the pair $\mu_{\Phi}, \Lambda_{\Phi}$, to each $\Phi \in \Xi$. The measure $\mu_{\Phi}$ is moreover concentrated on the Borel subset $\mathscr{F}$ of primary states of $\mathfrak{H}: \mu_{\Phi}(\mathscr{F})=\mu_{\Phi}(\mathfrak{\Xi})$ (the primary states of $\mathfrak{I}$ are those for which the weak closure of $\pi_{\Phi}(\mathfrak{U})$ is a factor in the sense of Von Neumann; they form a Borel set according to $[10,10 \mathrm{a}])$.

Remark. The kernel of the mapping $\Lambda_{\Phi}$ is the intersection of 3 and the kernel of $\pi_{\Phi}$. In other terms the statements $\Lambda_{\Phi}(Z)=0$ and $\pi_{\Phi}(Z)=0$, $Z \in 3$, are equivalent and, consequently, there is an isomorphism $\pi_{\Phi}(Z) \leftrightarrow \Lambda_{\Phi}(Z)$ between the center of $\pi_{\Phi}(\mathfrak{Q})^{\prime \prime}$ and the Von Neumann algebra $L^{\infty}\left(\Theta, \mu_{\Phi}\right): \Lambda_{\Phi}(Z)=0$ implies namely that $\Phi(Z A)=0$ for all $A \in \mathfrak{Q}$ and thus that $0=\Phi\left(A_{1} Z A_{2}\right)=\left(\Omega_{\Phi}\left|\pi_{\Phi}\left(A_{1}\right) \pi_{\Phi}(Z) \pi_{\Phi}\left(A_{2}\right)\right| \Omega_{\Phi}\right)$ for all $A_{1}, A_{2} \in \mathfrak{N}$ whence $\pi_{\Phi}(Z)=0$ by the cyclicity of $\Omega_{\Phi}$; and conversely $\pi_{\Phi}(Z)=0$ implies that $\Phi\left(Z^{*} Z\right)=\left\|\pi_{\Phi}(Z) \Omega_{\Phi}\right\|^{2}=0$ whence, setting $A=I$ in $(1),\left\langle\mu_{\Phi},\left|\Lambda_{\Phi}(Z)\right|^{2}\right\rangle=0$, whence $\Lambda_{\Phi}(Z)=0$.

Let now $g \in G \rightarrow \alpha_{g}$ be a homomorphism of the group $G$ into the automorphism group of $\mathfrak{2}\left(\alpha_{g_{1}^{-1} g_{2}}=\alpha_{g_{1}}^{-1} \cdot \alpha_{g_{2}}, g_{1}, g_{2} \in G\right)$. The isometric linear operator $\alpha_{g}, g \in G,[7 ; 1.8 .3]$ has a $w^{*}$-continuous isometric transposed $\alpha_{g}^{t}$ in $\mathfrak{Q}^{*}$ and a $\sigma$-continuous bitransposed $\alpha_{g}^{t t}$ in $\mathfrak{Q}^{* *}$. Further, since $\alpha_{g}$ is an automorphism of $\mathfrak{T}, \alpha_{g}^{t}$ leaves invariant the state space $\mathfrak{\subseteq} \leqq \mathfrak{I}^{*}$ (or, equivalently, acts on $\mathfrak{I}^{*}$ in an order-preserving manner) and $\alpha_{g}^{t t}$ is an automorphism of the Von Neumann algebra $\mathfrak{P}^{* *}$ [11; Lemma $(2,4)]$. On the other hand the mappings $g \in G \rightarrow \alpha_{g-1}^{t}$ and $g \in G \rightarrow \alpha_{g}^{t t}$ are group-homomorphisms. The following Lemma, which we do not claim to be original, gives equivalent formulations of the action of $G$ on $\mathfrak{2}$ or $\mathfrak{2} *$.

Lemma 1. Let $G$ be locally compact. The following are equivalent:

(i) $G$ acts on $\mathfrak{A}$ in a weakly continuous manner, i.e. $g \in G \rightarrow \Phi\left(\alpha_{g}(A)\right)$ is continuous (at $g=e$, the unit element of $G$ ) for all $\Phi \in \mathbb{S}\left(\Phi \in \mathfrak{Q}^{*}\right)$ and $A \in \mathfrak{I}$.

(ii) $G$ acts on $\mathfrak{R}$ in a strongly continuous manner i.e. $g \in G \rightarrow \alpha_{g}(A)$ is norm continuous (at $g=e$ ) for all $A \in \mathfrak{A}$.

(iii) The functions $g \in G \rightarrow \alpha_{g}^{t}(\Phi), \Phi \in \subseteq\left(\Phi \in \mathfrak{I}^{*}\right)$, are equicontinuous (at $g=e$ ). 
(iv) $G$ is a topological transformation group of $\subseteq$, i.e. the mapping

$$
(g, \Phi) \in G \times \Xi \rightarrow x_{g}^{t}(\Phi) \in \Xi
$$

is continuous from the topological product $G \times \Xi$ to $\Xi$.

Proof. Since $g \rightarrow \alpha_{g}$ is a group homomorphism, continuity properties at $g=e$ imply the same continuity properties at any spot in $G$. On the other hand condition (iii) and (iv) can be stated either for all $\Phi \in \Xi$ or equivalently for all $\Phi \in \mathfrak{U}^{*}$ since the latter are linear combinations of the former $[7 ; 2.6 .4]$. Condition (ii) obviously implies (i). The converse is a known fact in the theory of group representations on Banach spaces $[12 ; 10.2$ corollary $]$ and $[12 \mathrm{a}]$.

Condition (iii) on the other hand means that to each $g \in G$ and entourage $\mathscr{W}_{\varepsilon,\left\{A_{i}\right\}}=\left\{\left(\varphi_{1}, \varphi_{2}\right) \mid \varphi_{1}-\varphi_{2} \in \mathscr{V}_{\varepsilon .\left\{A_{i}\right\}}\right\}$ of $\Xi$ there is a neighbourhood $V$ of $g$ in $G$ such that $\left(\alpha_{g}^{t}(\Phi), \alpha_{g^{\prime}}^{t}(\Phi)\right) \in \mathscr{W}_{\varepsilon,\left\{A_{l}\right\}}$ for all $g^{\prime} \in V$ and $\Phi \in \Xi$. The last condition reads

$$
\begin{gathered}
\varepsilon>\operatorname{Sup}_{\Phi \in \mathbb{\Xi}}\left|\left\{\alpha_{g}^{t}(\Phi)-\alpha_{g^{\prime}}^{t}(\Phi)\right\}\left(A_{k}\right)\right|=\operatorname{Sup}_{\Phi \in \Xi}\left|\Phi\left(\alpha_{g}\left(A_{k}\right)-\alpha_{g^{\prime}}\left(A_{k}\right)\right)\right| \\
=\left\|\alpha_{g}\left(A_{k}\right)-\alpha_{g^{\prime}}\left(A_{k}\right)\right\|, \quad k=1,2, \ldots n,
\end{gathered}
$$

which shows the equivalence of (iii) and (ii). Now (iv) is obviously stronger than (i). On the other hand if we write

$$
\begin{gathered}
\left|\left\{\alpha_{g}^{t}(\Phi)-\alpha_{g^{\prime}}^{t}\left(\Phi^{\prime}\right)\right\}\left(A_{k}\right)\right|=\left|\left\{\alpha_{g}^{t}(\Phi)-\alpha_{g}^{t}\left(\Phi^{\prime}\right)\right\}\left(A_{k}\right)+\left\{\alpha_{g}^{t}\left(\Phi^{\prime}\right)-\alpha_{g^{\prime}}^{t}\left(\Phi^{\prime}\right)\right\}\left(A_{k}\right)\right| \\
\leqq\left|\left\{\Phi-\Phi^{\prime}\right\}\left(\alpha_{g}\left(A_{k}\right)\right)\right|+\left|\Phi^{\prime}\left(\alpha_{g}\left(A_{k}\right)-\alpha_{g^{\prime}}\left(A_{k}\right)\right)\right|
\end{gathered}
$$

we see that (iii) implies (iv).

\section{$\S$ 2. The Central Decomposition of Invariant States}

Our first result states the transformation properties of central measures and central homomorphisms under automorphisms of $\mathfrak{A}$.

Lemma 2. Let $\Phi \in \Xi$ and let $\alpha$ be an automorphism of $\mathfrak{U}$ with transposed $\alpha^{t}$ in $\mathfrak{U}^{*}$ and bitransposed $\alpha^{t t}$ in $\mathfrak{I}^{* *}$. The central measure of the state $\alpha^{t}(\Phi)$ is given by

$$
\left\langle\mu_{\alpha^{t}(\Phi)}, f\right\rangle=\left\langle\mu_{\Phi}, f \circ \alpha^{t}\right\rangle, \quad f \in \mathscr{C}(\Xi)
$$

$(\mathscr{C}(\mathbb{\Xi})$ is the set of continuous functions on $\mathbb{\Xi})$. For the corresponding central homomorphism one has

$$
\Lambda_{\gamma^{t}(\Phi)}(Z)=\left(\Lambda_{\Phi} \circ \alpha^{t t}\right)(Z)\left(\alpha^{t}\right)^{-1}, \quad Z \in 3 .
$$


In particular, for a state $\Phi$ invariant under $\alpha$ (i.e. such that $\left.\alpha^{t}(\Phi)=\Phi\right)$ the measure $\mu_{\Phi}$ is invariant under $\alpha^{t}$ :

$$
\left\langle\mu_{\Phi}, f \circ \alpha^{t}\right\rangle=\left\langle\mu_{\Phi}, f\right\rangle, \quad f \in \mathscr{C}(\Im)
$$

and the homomorphism $\Lambda_{\Phi}$ is such that

$$
\Lambda_{\Phi}(Z) \circ \alpha^{t}=\Lambda_{\Phi}\left(\alpha^{t t}(Z)\right), \quad Z \in \mathcal{3} .
$$

Proof. We have by (1) and by the definition of $\alpha^{t t}$, for $A \in \mathfrak{A}$ and $Z \in \mathbb{Z}$

$$
\begin{aligned}
\alpha^{t}(\Phi)(Z A) & =\Phi\left(\alpha^{t t}(Z) \alpha(A)\right) \\
& =\int_{\Xi} \Lambda_{\Phi}\left(\alpha^{t t}(Z)\right)(\psi) \cdot \psi(\alpha(A)) d \mu_{\Phi}(\psi) \\
& =\int_{\Phi}\left\{\left(\Lambda_{\Phi} \circ \alpha^{t t}\right)(Z) \circ \alpha^{t-1}\right\}\left(\alpha^{t}(\psi)\right) \cdot\left\{\alpha^{t}(\psi)\right\}(A) d \mu_{\Phi}(\psi)
\end{aligned}
$$

with $Z \rightarrow\left(\Lambda_{\Phi} \approx \alpha^{t t}\right)(Z) \circ \alpha^{t^{-1}}$ a $\sigma$-continuous homomorphism of 3 onto $L^{\infty}\left(\mathbb{S}, \mu_{\Phi}\right)$ due to the continuity of $\alpha^{t}$, the $\sigma$-continuity of $\alpha^{t t}$ and the automorphic property of the latter. Thus (6) is of the form (1) with $\Phi$ replaced by $\alpha^{t}(\Phi)$, so that (2) and (3) follow from the uniqueness of the central measure and central homomorphism associated with the state $\alpha^{t}(\Phi)$. Formulae (4) and (5) are specializations of (2) and (3) for states $\Phi$ such that $\alpha^{t}(\Phi)=\Phi$.

Corollary. Let $\Phi \in \subseteq$ with associated central measure $\mu_{\Phi}$ and let $\alpha$ be an automorphism of $\mathfrak{I}$ with transposed $\alpha^{t}$ in $\mathfrak{R}^{*}$. The state $\Phi$ is invariant under $\alpha$ (in the sense that $\Phi(\alpha(A))=\Phi(A)$ for all $A \in \mathfrak{V}$ ) if and only if the measure $\mu_{\Phi}$ is invariant under $\alpha^{t}$ (in the sense of fulfilling (4)).

Proof. If (4) holds we have from (1) where $Z=I$ and $A$ is arbitrary in $\mathfrak{A}$

$$
\begin{aligned}
\Phi(A) & =\int_{\Phi} \psi(A) d \mu_{\Phi}(\psi)=\int_{\mathscr{E}}\left\{\alpha^{t}(\psi)\right\}(A) d \mu_{\Phi}(\psi) \\
& =\int_{\mathscr{\Phi}} \psi(\alpha(A)) d \mu_{\Phi}(\psi)=\Phi(\alpha(A)),
\end{aligned}
$$

thus $\Phi$ is invariant. The converse is the statement that (4) results from $\alpha^{t}(\Phi)=\Phi$ in the previous Lemma.

Let us now return to the system $\{\mathfrak{H}, \alpha\}$ of a $C^{*}$-algebra $\mathfrak{A}$ and a homomorphism $g \in G \rightarrow \alpha_{g}$ of a group $G$ into the automorphism group of $\mathfrak{U}$. Let $\Phi$ be a $G$-invariant state of $\mathfrak{U}$, i.e. a state such that $\alpha_{g}^{t}(\Phi)=\Phi$ for all $g \in G$ (or else such that $\Phi\left(\alpha_{g}(A)\right)=\Phi(A)$ for all $g \in G$ and $A \in \mathfrak{A}$ ). In addition to the representation $\pi_{\Phi}$ of $\mathfrak{Q}, \Phi$ determines a unitary representation $U_{\Phi}$ of $G$ on the same Hilbert space with the properties $[13,7 ; 2.12 .11]$

$$
\begin{aligned}
& \pi_{\Phi}\left(\alpha_{g}(A)\right)=U_{\Phi}(g) \pi_{\Phi}(A) U_{\Phi}(g)^{-1} \\
& U_{\Phi}(g) \Omega_{\Phi}=\Omega_{\Phi}
\end{aligned}
$$


We will denote by $\pi_{\mathfrak{I}}$ and $U_{G}$ the respective sets $\left\{\pi_{\Phi}(A) \mid A \in \mathfrak{Q}\right\}$ and $\left\{U_{\Phi}(g) \mid g \in G\right\}$, by $\mathscr{R}$ the von Neumann algebra generated by $\pi_{\mathscr{R}}$ and $U_{G}$ and by $E_{0}$ the projector in $\mathscr{H}_{\Phi}$ onto the $U_{\Phi}$-invariant vectors. For a state $\Phi$ of $\mathfrak{A}$ invariant under $G$ one can consider the following different notions of "ergodicity" of which each implies the following (for the stated results we refer to e.g. [4]).

1) $E_{0}$ has rank one (equivalent to the "weak clustering property"

$$
M_{g}\left(\Phi\left(A \alpha_{g}(B)\right)=\Phi(A) \Phi(B) \text { for all } A, B \in \mathfrak{U}\right.
$$

where $M_{g}$ denotes the Godement mean).

2) $\Phi$ is extremal invariant (equivalent to $\mathscr{R}$ irreducible, or else $\left.\mathscr{R}^{\prime}=\pi_{\mathfrak{R}}^{\prime} \cap U_{G}^{\prime}=\{\lambda I\}\right)$.

3) $\mathscr{R}$ is a factor, or else $\mathscr{R} \cap \mathscr{R}^{\prime}=\mathscr{R} \cap \pi_{\mathfrak{H}}^{\prime} \cap U_{G}^{\prime}=\{\lambda I\}$.

4) $\pi_{\mathscr{H}}^{\prime \prime} \cap \pi_{\mathfrak{Q}}^{\prime} \cap U_{G}^{\prime}\left(=\pi_{\Phi}(3) \cap U_{G}^{\prime}\right)=\{\lambda I\}$.

Conversely one has the implication 4) $\Rightarrow 3$ ) in the case of a "large group of automorphisms" as defined by Störmer [17] (or more generally, see [4]); and 3) $\Rightarrow 2) \Rightarrow 1$ ) in the case of $G$-abelianness [2], that is if $E_{0} \pi_{21} E_{0}$ is abelian.

The preceding properties relate to the central decomposition of the state $\Phi$ through

Theorem 1. For a state $\Phi$ of $\mathfrak{A}$ invariant under $\alpha$, Property 4) above is equivalent to the ergodicity of the (invariant) central measure $\mu_{\Phi}$ of $\Phi$. In other terms the central measure $\mu_{\Phi}$ of $\Phi$ is ergodic if and only if the G-invariant elements in the center of $\pi_{\mathfrak{Q}}^{\prime \prime}$ are the multiples of the identity.

Proof. The ergodicity of $\mu_{\Phi}$ can be stated as the fact that the only $G$-invariant elements of $L^{\infty}\left(\Xi, \mu_{\Phi}\right)$ are the constant functions. Since the mapping $\Lambda_{\Phi}$ is onto, this is in turn equivalent to the requirement:

$$
\Lambda_{\Phi}(Z)=\alpha_{g}^{t}=\Lambda_{\Phi}(Z) \text { for some } Z \in 3 \text { and all } g \in G \Rightarrow \Lambda_{\Phi}(Z)=\lambda
$$

( $\lambda=$ some complex number). Using (5) we see that the left hand side of this implication is equivalent to $\Lambda_{\Phi}\left(\alpha_{g}^{t t}(Z)\right)=\Lambda_{\Phi}(Z)$ or to $\pi_{\Phi}\left(\alpha_{g}^{t t}(Z)\right)=\pi_{\Phi}(Z)$ whilst the right hand side means that $\pi_{\Phi}(Z)=\lambda I$ (cf. Remark in Section 1). Since we have $\pi_{\Phi}\left(\alpha_{g}^{t t}(Z)\right)=U_{\Phi}(g) \pi_{\Phi}(Z) U_{\Phi}\left(g^{-1}\right)$ the ergodicity of $\mu_{\Phi}$ is converted into the property:

$$
U_{\Phi}(g) \pi_{\Phi}(Z)=\pi_{\Phi}(Z) U_{\Phi}(g) \text { for some } Z \in 3 \text { and all } g \in G \Rightarrow \pi_{\Phi}(Z)
$$

is a scalar or else $\pi_{\Phi}(3) \cap U_{\Phi}(G)^{\prime}=\{\lambda I\}$. 
In view of the results stated above we then have the

Corollary. For an $\alpha$-invariant state $\Phi$ of a G-abelian system $\{\mathfrak{U}, \alpha\}$ (in particular, in the case of weak asymptotic abelianness) any of the "ergodic" properties 1) through 4) above is equivalent to the ergodicity of the central measure $\mu_{\Phi}$.

In the absence of an asymptotic abelian property we shall henceforth call an invariant state ergodic whenever its central measure is ergodic, i.e. if it fulfills property 4) above.

\section{§ 3. Transitive Ergodic States}

From now on we assume that $G$ is a locally compact separable ${ }^{2}$ group acting on our separable algebra with the continuity properties of Lemma 1. Furthermore we take the system $\{\mathfrak{U}, \alpha\}$ to be weakly asymptotically abelian [3, Definition 1]. Since the invariant states over such systems can be uniquely decomposed as integrals of ergodic states $[1,15]$, their investigation essentially reduces to that of ergodic states. Owing to Theorem 1 above, the latter is in turn converted into the study of the ergodic central measures, a problem in classical ergodic theory.

Generally, ergodic measures fall into two types, the transitive and intransitive type. In the present section we will discuss the single case of transitive central measures on $\mathbb{S}$, i.e. the measures concentrated on orbits. Specifically, given a $\varphi \in \Xi$, the orbit $\mathcal{O}_{\varphi}$ of $\varphi$ under $G$ is defined as the set $\left\{\alpha_{g}^{t}(\varphi) \mid g \in G\right\} . \mathcal{O}_{\varphi}$ is a Hausdorff space under the topology induced by $\Subset$ and the mapping $\psi, g \in \mathcal{O}_{\varphi} \times G \rightarrow \alpha_{g}^{t}(\psi) \in \mathcal{O}_{\varphi}$ is, owing to (iv) of Lemma 1 , continuous from the topological product $\mathcal{O}_{\varphi} \times G$ to $\mathcal{O}_{\varphi}$. Thus the stabilizer $S_{\varphi}$ of $\varphi$ (defined as the set of $g \in G$ such that $\alpha_{g}^{t}(\varphi)=\varphi$ ) is a closed subgroup of $G$ and the mapping $g \in G \rightarrow \alpha_{g}^{t}(\varphi) \in \mathcal{O}_{\varphi}$, constant on the left cosets module $S_{\varphi}$, determines a one-to-one continuous map of the left coset space $G / S_{\varphi}$ equipped with the quotient topology, into $\mathfrak{P}^{*}$ and onto the orbit $\mathcal{O}_{\varphi}[18 ; \S 7,19]$. Now if we equip the topological spaces under consideration with the Borel structures underlying their topologies, the homogeneous space $G / S_{\varphi}$ is, as $G$, locally compact with a denumerable basis of open sets and therefore standard $[23, \S 3]^{3}$. On the other hand the unit ball of the dual space $\mathfrak{U}^{*}$ of our separable $\mathfrak{U}$ is compact metrizable [7; B 7] and thus (complete and) standard. By [23; Theorem 3.2] (quoted in [7; B 21]) the one-to-one continuous and thus Borel mapping considered above has a Borel range $\mathcal{O}_{\varphi}$ and is Borel isomorphic from $G / S_{\varphi}$ onto $\mathcal{O}_{\varphi}$. We thus conclude that $\mathcal{O}_{\varphi}$ as a topological subspace of $\Xi$ and the homogeneous space $G / S_{\varphi}$ can be identified as Borel spaces.

\footnotetext{
${ }^{2}$ I.e. with a countable basis of open sets. In that case $G$ is metrizable.

${ }^{3} G / S_{\varphi}$ is polonais as an open set of its Alexandroff compactification which is metrizable complete of denumerable type $[18, \S 8.19][7, \mathrm{~B} 15]$.
} 
A regular measure $\mu$ on $\subseteq$ invariant under $G$ is now called transitive if there exists a $\varphi \in \mathbb{S}$ such that $\mu\left(\mathcal{O}_{\varphi}\right)=\mu(\mathbb{S})$ (or in other terms such that $\mathcal{O}_{\varphi}$ is of $\mu$-negligible complement - in that case $\mu$ is said to be concentrated on $\left.\mathcal{O}_{\varphi}\right)$. Transitive invariant measures are ergodic. Accordingly the $G$-invariant states of $\mathfrak{P}$ with transitive associated central measures are ergodic and we will call those states transitive. The transitive (invariant) ${ }^{4}$ states are the easiest type of ergodic states and they seem to be of wide occurrence in physics. Note that $\mu$ is a Radon measure on the homogeneous space $G / S_{\varphi}$ since $S_{\varphi}$ and $G / S_{\varphi}$ are Borel isomorphic and $G / S_{\varphi}$ is polonais [19; Proposition II-7-3].

Covariant Representations Generated by Transitive States. Let $\Phi$ be a transitive state with associated central measure $\mu_{\Phi}$ concentrated on the orbit $\mathcal{O}_{\varphi}$ of $\varphi \in \Xi$. The state $\varphi$ is by definition invariant under the subgroup $S_{\varphi}$ of $G$ and thus determines in the familiar way $[13,7 ; 2.12 .11]$ a covariant representation $\left(\pi_{\varphi}, L_{\varphi}\right)$ of the system $\left\{\mathfrak{H}, \alpha \mid S_{\varphi}\right\}$ where $\alpha \mid S_{\varphi}$ denotes the restriction of $\alpha$ to $S_{\varphi}$. We will now discuss the relationship of $\left(\pi_{\varphi}, L_{\varphi}\right)$ with the covariant representation $\left(\pi_{\Phi}, U_{\Phi}\right)$ of $\{\mathfrak{A}, \alpha\}$ determined by $\Phi$.

With $\pi_{\psi}$ the representation of $\mathfrak{A}$ (on the Hilbert space $\mathscr{H}_{\psi}$ ) determined by $\psi \in \mathbb{C}_{\varphi},\left\{\mathscr{H}_{\psi}\right\}_{\psi \in \mathbb{C}_{\varphi}}$ and $\left\{\pi_{\psi}\right\}_{\psi \in \mathscr{C}_{\varphi}}$ are respectively a Borel field of Hilbert spaces $^{5}$ and a Borel field of representations of $\mathfrak{A}$. Thus corresponding to the decomposition

$$
\Phi(A)=\int_{\mathscr{C}_{\varphi}} \psi(A) d \mu_{\Phi}(\psi), \quad A \in \mathfrak{A}
$$

we can define the following direct integrals

$$
\begin{aligned}
\mathscr{H} & =\int_{U_{\varphi}}^{\oplus} \mathscr{H}_{\psi} d \mu_{\Phi}(\psi), \\
\pi & =\int_{\mathscr{C}_{\varphi}}^{\oplus} \pi_{\psi} d \mu_{\Phi}(\psi)
\end{aligned}
$$

(these integrals extended to $\mathcal{O}_{\varphi}$ coincide with the same integrals extended to $\Xi$ since $\mu_{\Phi}$ is concentrated on $\mathcal{O}_{\varphi}$ ).

The representation $\pi$ defined by (8c) is unitarily equivalent to $\pi_{\Phi}$ via the unitary mapping $V$ from $\mathscr{H}_{\Phi}$ to $\mathscr{H}$ defined by

$$
V \hat{A}^{\Phi}=\int_{\mathscr{U}_{\varphi}}^{\oplus} \hat{A}^{\psi} d \mu_{\Phi}(\psi), \quad A \in \mathfrak{A},
$$

${ }^{4}$ The term "transitive state" implies that the state under consideration is invariant under $G$.

${ }^{5}$ For this notion see [7; A 96]. In our case the set $\Gamma$ of Borel vector fields consists of the vector fields $\left\{\xi_{\psi} \in \mathscr{H}_{\psi}\right\}_{\psi \in O_{\varphi}}$ such that the complex function $\psi \rightarrow\left(\xi_{\psi} \mid \hat{A}^{\psi}\right)$ is Borel for all $A \in \mathfrak{U}[6]\left(A_{\psi}=A \bmod \mathfrak{N}_{\psi}\right.$, see below). The field of representations $\left\{\pi_{\psi}\right\}_{\psi \in \mathcal{O}_{\varphi}}$ is Borel in the sense that $\left\{\pi_{\psi} \xi_{\psi}\right\}_{\psi \in \mathcal{O}_{\varphi}} \in \Gamma$ whenever $\left\{\xi_{\psi}\right\}_{\psi \in \mathcal{O}_{\varphi}} \in \Gamma[6]$. 
where $\hat{A}^{\Phi} \in \mathscr{H}_{\Phi}$ and $\hat{A}^{\psi} \in \mathscr{H}_{\psi}$ denote the classes of $A$ modulo the respective left ideals $\mathfrak{N}_{\Phi}$ and $\mathfrak{R}_{\psi}$ of $\Phi$ and $\psi\left(V\right.$ is isometric on $\mathfrak{X} / \mathfrak{N}_{\Phi}$ due to (8a) and thus extends to $\mathscr{H}_{\Phi}$ by continuity; the intertwining property $\pi V=V \pi_{\Phi}$ is immediately checked from the definitions of $\pi, \pi_{\psi}$ and $\pi_{\Phi}$; and $V$ is onto $\mathscr{H}$ due to the fact that $\mu_{\Phi}$ is a central measure as can be read off [5] - see also [6]). Further, if we identify $\pi_{\Phi}$ with $\pi,(8 \mathrm{c})$ is shown in [5, Theorem 2] to effect the central decomposition of $\pi_{\Phi} \cdot U_{\Phi}(g), g \in G$, is then identified with $U(g)=V U_{\Phi}(g) V^{-1}$, whose action on $\mathscr{H}$ is determined by

$$
U(g) \int_{\mathcal{U}_{\varphi}}^{\oplus} \hat{A}^{\psi} d \mu_{\Phi}(\psi)=\int_{U_{\varphi}}^{\oplus} \widehat{\alpha_{g}(A)^{\psi}} d \mu_{\Phi}(\psi), \quad A \in \mathfrak{A} .
$$

The preceding situation can be given an equivalent description using a direct integral of Hilbert spaces all of which coincide with $\mathscr{H}_{\varphi}$ : to this end we choose a regular Borel section $\psi \in \mathcal{O}_{\varphi} \rightarrow g(\psi) \in G$ of $G$ with respect to $S_{\varphi}$ in the sense of [20, Lemma 1.1]: $\psi \rightarrow g(\psi)$ is a Borel map of $\mathcal{O}_{\varphi}$ onto $G$ such that

$$
\alpha_{g(\psi)}^{t}(\varphi)=\psi^{6}, \quad \psi \in \mathcal{O}_{\varphi} ;
$$

and we define a unitary mapping $W_{\psi}$ from $\mathscr{H}_{\psi}$ to $\mathscr{H}_{\varphi}$ by

$$
W_{\psi} \hat{A}^{\psi}=\widehat{\alpha_{g(\psi)}(A)^{\varphi}}, \quad A \in \mathfrak{U}
$$

$\left(\hat{B}^{\psi}, \psi \in \mathcal{O}_{\varphi}\right.$, denotes as above the class of $B \in \mathfrak{A}$ modulo the left ideal $\mathfrak{N}_{\psi}$ of $\psi$. Since

$$
\begin{aligned}
\left\|\alpha_{g(\psi)}(A)\right\|_{\mathscr{H}_{\psi}}^{2} & =\varphi\left(\alpha_{g(\psi)}(A)^{*} \alpha_{g(\psi)}(A)\right)=\alpha_{g(\psi)}^{t}(\varphi)\left(A^{*} A\right) \\
& =\psi\left(A^{*} A\right)=\left\|\hat{A}^{\psi}\right\|_{\mathscr{H}_{\psi}}
\end{aligned}
$$

(12) defines an isometric linear mapping from $\mathfrak{I} / \mathfrak{P}_{\psi}$ onto $\mathfrak{Q} / \mathfrak{N}_{\varphi}$ whose continuous extension to $\mathscr{H}_{\psi}$ is thus unitary). To each vector field $\xi=\left\{\xi_{\psi} \in \mathscr{H}_{\psi}\right\}_{\psi \in \mathcal{U}_{\varphi}}$ we now associate the vector field $W \xi$ given by

$$
(W \xi)_{\psi}=W_{\psi} \xi_{\psi}
$$

with values in the constant Hilbert space $\mathscr{H}_{\varphi} \cdot W$ maps the set $\Gamma$ of Borel fields used for the definition of $\mathscr{H}$ onto the set of all Borel maps from $\mathcal{O}_{\varphi}$ to $\mathscr{H}_{\varphi}$ : since $W_{\psi}$ is unitary one has namely, for all fields $\xi$

$$
\left(\xi_{\psi} \mid \hat{A}^{\psi}\right)_{\mathscr{H}_{\psi}}=\left(W_{\psi} \xi_{\psi} \mid \widehat{\alpha_{g(\psi)}(A)^{\varphi}}\right)_{\mathscr{H}_{\varphi}}, \quad A \in \mathfrak{N},
$$

and one concludes from this that $\xi \in \Gamma$ holds if and only if $\psi \in \mathcal{O}_{\varphi}$ $\rightarrow\left(W_{\psi} \xi_{\psi} \mid \hat{A}^{\varphi}\right)_{\mathscr{H}_{\varphi}}$ is a Borel function for all $A \in \mathfrak{A}$ or equivalently if

${ }^{6} g(\psi)=B \cap \psi$ where $B$ is as in loc. cit. Lemma 1.1. where one takes left instead of right cosets and $\psi=\alpha_{g}^{t}(\varphi)$ is identified with the left coset $g S_{\varphi}$. The map $\psi \rightarrow g(\psi)$ is Borel due to [23, Theorem 4.2]. 
$\psi \in \mathcal{O}_{\varphi} \rightarrow\left(W_{\psi} \xi_{\psi} \mid u\right)_{\mathscr{H}_{\varphi}}$ is Borel for all $u \in \mathscr{H}_{\varphi}$. Furthermore

$$
\int_{\mathscr{C}_{\varphi}}\left\|\xi_{\psi}\right\|_{\mathscr{H}}^{2} d \mu_{\Phi}(\psi)=\int_{\mathcal{C}_{\varphi}}\left\|W_{\psi} \xi_{\psi}\right\|^{2} d \mu_{\Phi}(\psi)
$$

thus $W$ is a unitary mapping from $\mathscr{H}$ to the Hilbert space $L_{2}^{\mathscr{H}}{ }_{\varphi}\left(\mathcal{O}_{\varphi}, \mu_{\Phi}\right)$ of square-integrable maps from $\mathscr{O}_{\varphi}$ to $\mathscr{H}_{\varphi}$. Transporting via $W$ the covariant representation $(\pi, U)$ of $(8 \mathrm{c})$ and $(10)$ one gets operators acting on $\eta \in L^{\mathscr{H} \varphi}\left(\mathcal{O}_{\varphi}, \mu_{\Phi}\right)$ in the following manner

$$
\begin{gathered}
\left(W \pi(B) W^{-1} \eta\right)_{\psi}=\pi_{\varphi}\left(\alpha_{g(\psi)}(B)\right)_{\eta_{\psi}}, \quad B \in \mathfrak{A}, \\
\left(W U(s) W^{-1} \eta\right)_{\psi}=L_{\varphi}\left(g(\psi) s g\left(\alpha_{s}(\psi)\right)^{-1}\right) \eta_{\alpha_{s}(\psi)}, \quad s \in G .
\end{gathered}
$$

It is enough to check those formulae on the dense set of elements of the form

$$
\eta=W \int_{\mathscr{O}}^{\oplus} \hat{A}^{\psi} d \mu_{\Phi}(\psi), \quad A \in \mathfrak{N},
$$

for which $(8 \mathrm{c})$ and $(10)$ give

whence by (12)

$$
\begin{aligned}
& \left(\pi(B) W^{-1} \eta\right)_{\psi}=\pi_{\psi}(B) \hat{A}^{\psi}=\widehat{B A}^{\psi} \\
& \left(U(S) W^{-1} \eta\right)_{\psi}={\widehat{\alpha_{s}}(A)^{\psi}}^{\psi}
\end{aligned}
$$

$$
\begin{aligned}
& \left(W \pi(B) W^{-1} \eta\right)_{\psi}=\widehat{\alpha_{g(\psi)}(B A)^{\varphi}} \\
& \left(W U(s) W^{-1} \eta\right)_{\psi}=\widehat{\alpha_{g(\psi) s}(A)^{\varphi}}
\end{aligned}
$$

from which $(15 \mathrm{a}, \mathrm{b})$ follow using the definition of $\pi_{\varphi}$ and $L_{\varphi}$ and the fact that $g(\psi) s g\left(\alpha_{s}(\psi)\right) \in S_{\varphi}$ owing to (11).

Formulae $(15 \mathrm{a}, \mathrm{b})$ express the fact that $\left(\pi_{\Phi}, U_{\Phi}\right)$ is unitarily equivalent to the covariant representation of $\{\mathfrak{U}, \alpha\}$ induced by $\left(\pi_{\psi}, L_{\psi}\right)$ in the sense defined by Fell, Zeller-Meier, and Takesaki in Refs. [25] through [28]. We will now show this in a more direct way making contact with [28, Definition 3.2]. As is well known an important class of group representations can be described following Mackey as induced by a subgroup (Refs. [20] through [22]). A natural generalization of Mackey's theory allows to describe in an analogous way a wide class of covariant representations of a system $\{\mathfrak{R}, \alpha\}$ as induced by representations "with lower symmetry" 7 , i.e. covariant only with respect to a subgroup (Refs. [24] through [28]). Specifically, the representation $(\tilde{\pi}, \tilde{U})$ of $\{\mathfrak{H}, \alpha\}$ induced by $\left(\pi_{\varphi}, L_{\varphi}\right)$ is defined as follows:

(i) the corresponding Hilbert space $\tilde{\mathscr{H}}$ is that of Borel maps $\eta_{g}: g \rightarrow \eta_{g}$ from $G$ to $\mathscr{H}_{\varphi}$ fulfilling the identity ${ }^{8}$

$$
\eta_{\gamma g}=L_{\varphi}(\gamma) \eta_{g}, \quad g \in G, \quad \gamma \in S_{\varphi},
$$

7 Or "with spontaneously broken symmetry" in the physicist's terminology.

${ }^{\gamma}$ In what follows we use the notation $f(\hat{g})$ for the function $g \in G \rightarrow f(g)$, the roofed letter $\hat{g}$ indicating a dummy variable 
and square integrable in the sense that

$$
\left\|\eta_{\dot{g}}\right\|_{\dot{\mathscr{H}}}=\int\left\|\eta_{g}\right\|_{\mathscr{H}_{\varphi}} d \mu_{\Phi}(\psi)<\infty
$$

(here $\psi=\alpha_{g}^{t}(\varphi)$; due to (16) the norm $\left\|\eta_{g}\right\|_{\mathscr{H}_{\varphi}}$ of $\eta_{\dot{g}}$ in $\tilde{\mathscr{H}}$ depends only upon the left $\operatorname{coset} \psi=g S_{\varphi} ;(17)$ is shown to define a Hilbert space norm).

(ii) $\tilde{U}$ is the representation of $G$ induced by $S_{\varphi}$ :

(iii) One has

$$
(\tilde{U}(s) \eta)_{g}=\eta_{g s}, \quad g, s \in G .
$$

$$
(\tilde{\pi}(A) \eta)_{g}=\pi_{\varphi}\left(\alpha_{g}(A)\right) \eta_{g}, \quad A \in \mathfrak{N}, g \in G .
$$

We first set up a unitary map $S$ from $\mathscr{H}_{\Phi}$ to $\tilde{\mathscr{H}}$ by defining

$$
S \pi_{\Phi}(Z) \hat{A}^{\Phi}=\Lambda_{\Phi}(Z)\left(\alpha_{\hat{g}}^{t}(\varphi)\right) \widehat{\alpha_{g}(A)^{\varphi}}, \quad A \in \mathfrak{A}, Z \in \mathcal{B} .
$$

Let us check that the right hand side of this equation is an element of $\tilde{\mathscr{H}}$. First it is a Borel map from $G$ to $\mathscr{H}_{\varphi}$ since $\alpha_{\tilde{g}}^{t}(\varphi)$ is continuous, and thus Borel, from $G$ to $\Xi ; \Lambda_{\Phi}(Z) \in L^{\infty}\left(\Theta, \mu_{\Phi}\right)$ can be chosen to be a Borel function; and $\widehat{\alpha_{i j}(A)}$ is continuous, and thus Borel, from $G$ to $\mathscr{H}_{\varphi}$ due to the assumed property (ii) of Lemma 1 . On the other hand property (16) is fulfilled since, for all $A \in \mathfrak{R}, Z \in \mathfrak{B}, g \in G$ and $\gamma \in S_{\varphi}$, using the definitions of $S_{\varphi}$ and $L_{\varphi}$,

$$
\Lambda_{\Phi}(Z)\left(\alpha_{; g}^{t}(\varphi)\right) \widehat{\alpha_{j g}(A)^{\varphi}}=\Lambda_{\Phi}(Z)\left(\alpha_{g}^{t}(\varphi)\right) L_{\varphi}(\gamma) \widehat{\alpha_{g}(A)^{\varphi}} .
$$

Finally, we have, by (1)

$$
\begin{aligned}
\left\|\Lambda_{\Phi}(Z)\left(\alpha_{\hat{g}}^{t}(\varphi)\right) \widehat{\alpha_{g}(A)^{\varphi}}\right\|_{\mathscr{\mathscr { H }}}^{2} & =\int_{\mathscr{C}_{\varphi}} \Lambda_{\Phi}\left(Z^{*} Z\right)\left(\alpha_{g}^{t}(\varphi)\right) \varphi\left(\alpha_{g}\left(A^{*} A\right)\right) d \mu_{\Phi}(\psi) \\
& =\Phi\left(Z^{*} Z A^{*} A\right)=\Phi(Z A)^{*}(Z A)=\left\|\pi_{\Phi}(Z) \hat{A}^{\Phi}\right\|_{\mathscr{H} \Phi}
\end{aligned}
$$

and thus (20) defines $S$ as an isometric linear mapping from $\mathfrak{A} / \mathfrak{M}_{\Phi}$ into $\tilde{\mathscr{H}}$ which extends to $\mathscr{H}_{\Phi}$ by continuity. To show that $S$ is onto $\tilde{\mathscr{H}}$ it suffices to check that the set $C$ of elements of the form (20) where $A$ runs through $\mathfrak{A}$ and $Z$ through 3 , is total in $\tilde{\mathscr{H}}$. But this follows from [20, Lemma 3.3]: condition (a) of this Lemma has already been verified; condition (b) holds with $\varrho_{s}=1$ because, due to (5)

$$
\begin{aligned}
\Lambda_{\Phi}(Z)\left(\alpha_{g s}^{t}(\varphi)\right) \widehat{\alpha_{g s}(A)^{\varphi}} & =\Lambda_{\Phi}\left(\alpha_{s}^{t t}(Z)\right)\left(\alpha_{g}^{t}(\varphi)\right) \widehat{\alpha_{g}\left(\alpha_{s}(A)\right)^{\varphi}} \\
& =S \pi_{\Phi}\left(\alpha_{s}^{t t}(Z)\right) \widehat{\alpha_{s}(A)^{\varphi}} ;
\end{aligned}
$$

condition (c) follows from the fact that $\Lambda_{\Phi}$ is onto $L_{\infty}\left(\mathfrak{S}, \mu_{\Phi}\right)$; and condition (d) from the separability of $\mathfrak{A}$.

The identification of $\left(S \pi_{\phi} S^{-1}, S U_{\Phi} S^{-1}\right)$ as the covariant representation $(\tilde{\pi}, \tilde{U})$ of $\{\mathfrak{N}, \alpha\}$ induced by $\left(\pi_{\varphi}, L_{\varphi}\right)$ now reduces to a straightforward 
verification: we have, for all $B \in \mathfrak{A}$

$$
\begin{gathered}
S \pi_{\Phi}(B) S^{-1} \cdot S \pi_{\Phi}(Z) \hat{A}^{\Phi}=S \pi_{\Phi}(Z) \widehat{B A}^{\Phi} \\
=\Lambda_{\Phi}(Z)\left(\alpha_{\dot{g}}^{t}(\varphi)\right) \widehat{\alpha_{g}(B A)^{\Phi}}=\pi\left(\alpha_{g}(B)\right) A_{\Phi}(Z)\left(\alpha_{\dot{g}}^{t}(\varphi)\right) \widehat{\alpha_{\dot{g}}(A)^{\varphi}}
\end{gathered}
$$

and, for all $s \in G$, by (5)

$$
\begin{gathered}
S U_{\Phi}(s) S^{-1} \cdot S \pi_{\Phi}(Z) \hat{A}^{\Phi}=S \pi_{\Phi}\left(\alpha_{s}^{t t}(Z)\right) \widehat{\alpha_{s}(A)^{\Phi}} \\
=\Lambda_{\Phi}\left(\alpha_{s}^{t t}(Z)\right)\left(\alpha_{\hat{g}}^{t}(\varphi)\right) \widehat{\alpha_{\hat{g}}\left(\alpha_{s}(A)\right)^{\varphi}}=A_{\Phi}(Z)\left(\alpha_{\hat{g} s}^{t}(\varphi)\right) \widehat{\alpha_{g s}(A)^{\Phi}}
\end{gathered}
$$

which checks with (18) and (19) as applied to elements of the total set (20).

We subsume the results of this section in the

Theorem 2. Assume that $\alpha$ is strongly continuous in the sense of (ii) Lemma 1 and let $\Phi$ be a transitive (invariant) state of $\{\mathfrak{H}, \alpha\}$, i.e. such that the associated central measure $\mu_{\Phi}$ is concentrated on the orbit

$$
\mathcal{O}_{\varphi}=\left\{\alpha_{g}^{t}(\varphi) \mid g \in G\right\}
$$

of $\varphi \in \mathbb{\Xi}$ under $G$. The canonical mapping of $G / S_{\varphi}$, with

$$
S_{\varphi}=\left\{g \in G \mid \alpha_{g}^{t}(\varphi)=\varphi\right\},
$$

the stabilizer of $\varphi$, onto $\mathcal{O}_{\varphi}$ equipped with the $w^{*}$-topology, is a Borel isomorphism. The covariant representation $\left(\pi_{\Phi}, U_{\Phi}\right)$ of $\{\mathfrak{A}, \alpha\}$ generated by $\Phi$ is unitarily equivalent to the covariant representation $(\tilde{\pi}, \tilde{U})$ induced by $\left(\pi_{\varphi}, L_{\varphi}\right)$ [see (18) and (19)], where $\left(\pi_{\varphi}, L_{\varphi}\right)$ is the covariant representation of $\left\{\mathfrak{N}, \alpha \mid S_{\varphi}\right\}$ generated by $\varphi$. Other unitarily equivalent versions are given by the direct integrals $\pi$ and $U$ defined in $(8 \mathrm{c})$ and (10) on the direct integral Hilbert space (8b), where (8c) is the central decomposition of $\pi$; or by the operators $(15 \mathrm{a}, \mathrm{b})$ on the Hilbert space $L^{\mathscr{H}} \varphi\left(\mathcal{O}_{\varphi}, \mu_{\Phi}\right)$ of $\mu_{\Phi}$-square integrable maps from $\mathcal{O}_{\varphi}$ to $\mathscr{H}_{\varphi}$.

\section{$\S$ 4. Ergodicity of States with Respect to Time Translations. Stability and Primariness}

As a first physical application of the foregoing study we discuss in this section ergodicity with respect to time translations. Thus $\mathfrak{A}$ is now the quasi-local algebra of field theory, $G$ the group of additive reals and we assume that

i) time translations induce automorphisms $t \rightarrow \alpha_{t}$ of $\mathfrak{Q}$.

ii) The continuity property of Lemma 1 above (which we hope can be achieved by a proper choice of $\mathfrak{Q})$. 
iii) Asymptotic abelianness in the weak sense, i.e.

$$
\Phi\left(\left[A, \alpha_{t}(B)\right]\right) \underset{t=\infty}{\longrightarrow} 0
$$

for each state $\Phi$ of $\mathfrak{Q}$ and elements $A, B \in \mathfrak{U}$ ([ ] denotes a commutator).

The results of the foregoing section relative to transitive ergodic states now assume a particularly simple form: due to the abelianness of $G$ (as would be the case more generally whenever the stabilizer $S_{\varphi(p)}$ associated to the state $\Phi$ under consideration is a normal subgroup of $G$ ), the quotient $G / S_{\varphi}$ is a group and therefore, as equipped with a bounded Haar measure, compact. Now a compact quotient group of the reals is either a one-dimensional torus or reduces to a point. The first case corresponds to a transitive ergodic state of the form

$$
\Phi=\int_{0}^{T} \alpha_{,}^{t}(\psi) d s,
$$

where $d s$ denotes Lebesgue measure and $\psi$ is a primary state of (smallest) time period $T$ :

$$
\alpha_{s+T}^{t}(\psi)=\alpha_{s}^{t}(\psi), \quad s \in \mathbb{R} .
$$

In the second case $S_{\varphi}$ is the whole group $G, \mathcal{O}_{\varphi}=\{\varphi\}, \pi \Phi$ is the Dirac measure concentrated at $\varphi=\Phi$ and our invariant ergodic state is itself primary. In this case $\Phi$ is not only ergodic but, as known since long [1], strongly clustering in the sense that

$$
\Phi\left(A \alpha_{t}(B)\right)-\Phi(A) \Phi\left(\alpha_{t}(B)\right) \underset{t=\infty}{\longrightarrow} 0
$$

for all $A, B \in \mathfrak{A}$ (this holds in fact for primary states wether invariant or not, as we shall need below).

Our claim is now that the only transitive ergodic states ( for the time evolution) encountered in physics as simple thermodynamic phases are of the latter type, and thus primary. We will infer this from the assumption that one-phase equilibrium states should possess the following property of $\mathfrak{Y}$-stability.

Definition. Let $\mathfrak{I}$ be a $C^{*}$-algebra with a one-parameter group of automorphisms $t \rightarrow \alpha_{t}$. A state $\Phi$ of $\mathfrak{Q}$ is called $\mathfrak{A}$-stable whenever

$$
\alpha_{t}^{t}\left[\Phi_{A}-\Phi\left(A^{*} A\right) \cdot \Phi\right] \underset{t=\infty}{\longrightarrow} 0
$$

in the $w^{*}$-topology, for all elements $A \in \mathfrak{A}$ (we here use the notation $\left.\Phi_{A}(X)=\Phi\left(A^{*} X A\right), X \in \mathfrak{V}\right)$.

The cogency of this assumption rests upon the view that the operation $\Phi \rightarrow \Phi_{A}$ corresponds physically (apart from a normalization factor) to a local perturbation of the state $\Phi$, if $A$ is local; and that local perturbations applied to genuinely stable (not metastable) states should be evanescent 
in time. (As is evident from continuity, it is immaterial wether we state the above definition for strictly local rather than for general elements $A$ of $\mathfrak{Q})$.

Our claim follows from the assumption of $\mathfrak{Q}$-stability by means of the

Proposition. With the assumptions stated in the beginning of this section, let $\Phi$ be a transitive ergodic state of the form (21). If, for $A \in \mathfrak{A}$, the function $t \rightarrow \Phi\left(A^{*} \alpha_{t}\left(A^{*} A\right) A\right)$ admits a limit for $t=+\infty$, the function $t \rightarrow y\left(\alpha_{t}\left(A^{*} A\right)\right)$ is constant.

Proof. Suppose that $\Phi\left(A^{*} \alpha_{t}\left(A^{*} A\right) A\right) \underset{t=+\infty}{\longrightarrow} L$ then, by assumption iii) above and the fact that bounded linear forms are linear combinations of states,

$$
\Phi\left(A^{*} A \alpha_{t}\left(A^{*} A\right)\right)=\int_{s=0}^{T}\left\{\alpha_{s}^{t}(\psi)\right\}\left(A^{*} A \alpha_{t}\left(A^{*} A\right)\right) d s \underset{t=\infty}{\longrightarrow} L .
$$

Now, since the state $\alpha_{s}^{t}(\psi)$ is primary and thus strongly clustering the function

$$
t \rightarrow\left\{\alpha_{s}^{t}(\psi)\right\}\left(A^{*} A \alpha_{t}\left(A^{*} A\right)\right)-\psi\left(\alpha_{s}\left(A^{*} A\right)\right) \cdot \psi\left(\alpha_{s+1}\left(A^{*} A\right)\right)
$$

tends to zero for $t=+\infty$ and remains bounded by $2 \| A^{*} A^{\| 2}$.

Thus, setting $u(s)=\psi\left(\alpha_{s}\left(A^{*} A\right)\right)$, the dominated convergence theorem permits to deduce from (23) that

$$
v(-t)=\int_{s=0}^{T} u(s) u(s+t) d s
$$

tends towards $L$ as $t \rightarrow+\infty$. Now $u$, and thus $v$, are periodic with period $T$. Therefore $v(t)=L$ for all $t$, and if one denotes by $u_{n}$ and $v_{n}$ the respective Fourier coefficients of the functions $u$ and $v$ on the circle. it follows that $v_{n}=\left|u_{n}\right|^{2}=0$ for all $n \neq 0$. Thus $u(t)$ is independent of $t$, completing the proof.

It is now straightforward that $\mathfrak{P}$-stable transitive ergodic states must be primary. A non primary transitive state $\Phi$, thus of the form (1), would namely, if $\mathfrak{Q}$-stable, be such that $t \rightarrow \Phi\left(A^{*} \alpha_{t}\left(A^{*} A\right) A\right)$ would admit a limit at $t=+\infty$ for every $A \in \mathfrak{I}$, implying by the last proposition that the state $\psi$ would not depend upon time. This would contradict the assumption that the orbit of $\psi$ is as a set a one-dimensional torus. Transitive ergodicity and $\mathfrak{A}$-stability thus imply primariness. Reversely primariness implies $\mathfrak{Y}$-stability as follows from strong clustering and asymptotic abelianness as stated under iii) above.

We leave open the question of wether $\mathfrak{A}$-stable intransitive ergodic states are automatically primary under our assumptions. We conjecture that this is the case. 


\section{§ 5. Transitive Euclidean States}

5.1.

In this section, we characterize all the symmetry groups (= stabilizers) of transitive states for the connected euclidean group $E^{9}$. We have shown that such a symmetry group $H$ is, up to a conjugation, a closed subgroup of $E$ for which the associated homogeneous space $E / H$ carries a finite $E$-invariant measure. Our classification will of course yield the crystallographic groups in three dimensions, the groups obtained from the one and two dimensional crystallographic groups and the closed subgroups of the rotation group. But we will also find another infinite family of groups in addition.

In 5.2 we will recall some properties of the Euclidean group E. In 5.3 we will classify the conjugacy classes of closed subgroups of $E$; in 5.4 we shall divide our problem into a few different cases to be studied separately.

We end this section by listing some mathematical results useful for our problem. For general references, see e.g. [30, 33, 34].

Let $G$ be a locally compact group. Its modular function $\Delta_{G}$ is a continuous homomorphism from $G$ to $R^{\times}$, the multiplicative group of positive real numbers (see [33], $\$ 1.3 ;[34], 15.11$ ). Abelian or compact or discrete groups are unimodular (i.e. $\forall g \in G, \Delta_{G}(g)=1$ ). Groups which have no non trivial abelian homomorphic image are also unimodular. This is the case of the Euclidean group $E$.

Let $A, B$ be closed subgroups of $G$ locally compact and $A \subset B \subset G$.

(i) The homogeneous space $G / A$ is a topological quotient and moreover, because of the group law, the canonical continuous map $G \stackrel{\uplus}{\rightarrow} A$ is open (image of open sets are open). $G / A$ is locally compact ([34], 5.22). If $A$ is compact, $\psi$ is also a closed mapping (the image of a closed set is closed) ([34], 5.18). If both $A$ and $G / A$ are compact, then $G$ is compact ([34], 5.25).

(ii) If $A$ is an invariant subgroup of $G$, we denote by $\varphi$ the canonical homomorphism of topological groups $G \stackrel{\varphi}{\rightarrow} G / A$. Then $B / A$ is topologically isomorphic (i.e. isomorphic as topological group) to the subgroup $\varphi(B)$ of $G / A$; we write $B / A \approx \varphi(B)([33] \S 2.7$, Prop. 20, [34] 5.31). Furthermore the homogeneous spaces $G / B$ and $(G / A) /(B / A)$ are homeomorphic: we write it $G / B \sim(G / A) /(B / A)$ (see [33] $\$ 2.8$, Prop. 22).

(iii) If $G, A, B$ are unimodular, $G / A$ and $G / B$ carry $G$-invariant measures (unique up to a factor) denoted respectively $m_{A}$ and $m_{B}$. The homogeneous space $B / A$ carries a $B$-invariant measure $m^{\prime}$ such that

\footnotetext{
${ }_{9}$ The case of the physically relevant full Euclidean group (including the space symmetries) can trivially be adapted from our results.
} 
$m_{A}=m_{B} \otimes m^{\prime}$ so that one has for the total measures

$$
m_{A}(G / A)=m_{B}(G / B) \cdot m^{\prime}(B / A) .
$$

We will use repeatedly the more precise Lemma (Mostow [29], Lemma 2.5, Bourbaki [30], §2, n 8 corollaire 2):

Lemma 4. Let $G$ be a locally compact group and $A \subset B$ closed subgroups of $G$. If $G / A$ has a finite $G$-invariant measure $m_{A}$, both $G / B$ and $B / A$ admit finite invariant measures $m_{B}$ and $m^{\prime}$ of which $m_{A}$ is a product.

(iv) If $G / A$ carries a $G$-invariant measure and is compact, $m_{A}(G / A)$ is finite. The converse is not true in general. However if $A$ is an invariant subgroup and $G / A$ carries a $G$-invariant measure $m_{A}$ its finiteness implies the compactness of the group $G / A$.

Mostow has also shown [29]:

Theorem. When $G$ is a solvable Lie group, for any closed subgroup $A$ of $G, m_{A}$ invariant finite implies $G / A$ compact.

This is also true when $G$ is the extension of a compact Lie group by a solvable group. (Mostow's private communication). The euclidean group $E$ is of this type; our work verifies that it satisfies this extension of Mostow's theorem.

We conclude this $\S 5.1$ with three important strategical remarks. Let $H$ be a solution of the problem for $E$, i.e. $H$ is a closed subgroup of $E$ such that the homogeneous space $E / H$ carries a bounded $E$-invariant measure:

1) All closed subgroups $H^{\prime}$ of $E$ such that $H \subset H^{\prime} \subset E$ are solutions of the same problem; this is due to Lemma 4.

2) All subgroups $H^{\prime \prime} \mathrm{CH}$ solutions of the problem for $H$ are solutions of the problem for $E$.

Indeed, since $E$ is unimodular, $H$ and $H^{\prime \prime}$ are also unimodular, so $E / H^{\prime \prime}$ carries an $E$-invariant measure and from (24)

$$
m_{H^{\prime \prime}}\left(E / H^{\prime \prime}\right)=m_{H}(E / H) \cdot m^{\prime}\left(H / H^{\prime \prime}\right)
$$

so the finiteness of $m_{H}(E / H)$ and of $m^{\prime}\left(H / H^{\prime \prime}\right)$ implies that of $m_{H^{\prime \prime}}\left(E / H^{\prime \prime}\right)$.

3) Conversely if $K$ is not a solution of the problem for $H$, it is not a solution for $E$.

\subsection{Properties of the Euclidean Group}

We recall that the Euclidean group $E=T \square K$ is the semi-direct product of $K$, the connected real orthogonal group $S O$ (3), by the abelian group $T$ of three dimensional translations. We shall denote $(t, r), t \in T$, 
$r \in K$. an element of $E$. The group multiplication law is

$$
(t, r)\left(t^{\prime}, r^{\prime}\right)=\left(t+r \cdot t^{\prime}, r r^{\prime}\right)
$$

where $r \cdot t^{\prime}$ is the transformed of $t^{\prime}$ in the orthogonal transformation $r$, i.e.

$$
(0, r)\left(t^{\prime}, 1\right)\left(0, r^{-1}\right)=\left(r \cdot t^{\prime}, 1\right) .
$$

The elements $(0, r), r \in K$, form a subgroup $K(0)$ of $E$ which is isomorphic to $K$.

By recursion, one proves:

$$
(a, r)^{n}=\left(\left(\sum_{k=0}^{n-1} r^{k}\right) \cdot a, r^{n}\right) .
$$

We shall need the value of the commutator of two elements

$(a, r)(b, s)(a, r)^{-1}(b, s)^{-1}=\left(\left(1-r s r^{-1}\right) a+r\left(1-s r^{-1} s^{-1}\right) b, r s r^{-1} s^{-1}\right)$.

We denote by $\psi$ and $\varphi$ the continuous map $T \stackrel{\varphi}{\leftarrow} E \stackrel{\varphi}{\longrightarrow} K=E / T$

$$
\psi(t, r)=t, \quad \varphi(t, r)=r .
$$

We remark (see (i)) that $\psi$ is a closed map and $\varphi$ a homomorphism of topological groups. We also need some remarks on the compact subgroups of $E$. Such a subgroup $C$ cannot contain a non zero translation $(t, 0)$ because it would have to contain all translations: $n \in Z,(n t, 0)$ and $C \cap T$ would not be compact. Then $C$ cannot have two distincts elements with same $r$, indeed $(t, r)\left(t^{\prime}, r\right)^{-1}=\left(t-t^{\prime}, 1\right)$ is a translation. So the elements of $C$ are of the form $(\tau(r), r)$ where $\tau:\left.\varphi\right|_{C}=\left.\psi\right|_{C}$ is a continuous map $C \stackrel{\tau}{\rightarrow} T$. The associativity of the group law requires

$$
\tau\left(r_{1}\right)+r_{1} \cdot \tau\left(r_{2}\right)=\tau\left(r_{1} r_{2}\right) .
$$

Since $C \cap T=\{0\}, C \approx \varphi(C) \subset K$. (If $C$ were not compact the isomorphism would not be topological.) One proves that all $\tau$ which satisfy (27) are of the form

$$
\tau(r)=(1-r) a
$$

with a fixed element $a \in T$; so

$$
(\tau(r), r)=(a, 1)(0, r)(a, 1)^{-1} \in K(a) .
$$

Every maximal compact subgroup of $E$ is conjugated to $K(0)$.

A compact group $C$ is unimodular, so the homogeneous space $E / C$ has a $E$-invariant measure $m_{l}$, and is not compact (since $E$ is not compact, see (i)). We prove that $m_{i}(E, C)$ is not finite: indeed all $E / K(t)$ are isomorphic (as homogeneous spaces) to $E / K(0)=T$ on which the $E$-invariant 
measure is the Lebesgue measure $m_{K}$; it is not finite on $T$ and from (24)

$$
m(E / C)=m_{K}(E / K(0)) \cdot m^{\prime}(K(0) / C)
$$

hence $m_{C}(E / C)$ is not finite.

\subsection{The Closed Subgroups of $E$}

Let $H$ be a closed subgroup of $E$. Then

$$
H \cap T=T_{H}
$$

is a closed subgroup of $T$ and an invariant subgroup of $H$.

The list of closed subgroups of $T$, up to an automorphism of $T$ is well-known (see e.g. [31], §1, n 2):

$$
\begin{gathered}
\{0\}, Z, R, Z^{2}, Z+R, R^{2}, \\
Z^{3}, Z^{2}+R, Z+R^{2}, R^{3}
\end{gathered}
$$

where we have denoted respectively $R$ and $Z$ the additive group of real numbers and integers. We also use the notations $R^{k}$ or $Z^{p}$ for the direct sum of $k$ or $p$ groups isomorphic to $R$ or $Z$. For instance the translation group $T$ is isomorphic to $R^{3}$.

We will need also to know the normalizer in $E$ of these closed subgroups $T_{H} \subset T: N(G)$, the normalizer of the subgroup $G$ of $E$ is the set $\left\{g \in E, g G g^{-1} \cong G\right\}$, i.e. $N(G)$ is the largest subgroup of $E$ which contains $G$ as invariant subgroup. When $G=T_{H} \subset T$, then $T \subset N\left(T_{H}\right)$ since $T$ is abelian. Elementary geometrical considerations, based on equation (25) lead to the computation of $N\left(T_{H}\right)$ for the different closed subgroups of $T$ in (29) and $\left(29^{\prime}\right)$. We list the corresponding quotient groups $Q_{H}=N\left(T_{H}\right) / T$ in Table 1. Note that $N\left(T_{H}\right)$ is the semi-direct product of $Q_{H}$ by $T$.

Let us denote by $K_{H}$ the topological quotient group $H / T_{H}$ :

$$
K_{H}=H / T_{H} \text {. }
$$

Table 1

\begin{tabular}{llllllll}
\hline a) & $T_{H}$ & $\{0\}$ & $Z$ & $R$ & $Z^{2}$ & $Z+R$ & $R^{2}$ \\
& $Q_{H}$ & $K=S O(3)$ & $O(2)^{a}$ & $O(2)^{a}$ & finite & finite & $O(2)^{a}$ \\
\hline b) & $T_{H}$ & $\begin{array}{l}Z^{3} \\
\text { finite }\end{array}$ & $\begin{array}{l}Z^{2}+R \\
\text { finite }\end{array}$ & $\begin{array}{l}Z+R^{2} \\
O(2)^{\mathrm{a}}\end{array}$ & $\begin{array}{l}R^{3}=T \\
K=S O(3)\end{array}$ & & \\
\hline
\end{tabular}

a The group $Q_{H}$ isomorphic to $O(2)$ contains the rotations around an axis and the rotations of $\pi$ around perpendicular axes. 
From (28), (30), (i), there is a natural continuous injective group homomorphism:

$$
K_{H} \stackrel{\leftrightarrow}{\rightarrow} Q_{H} .
$$

Note that $i$ is not in general a closed mapping.

We recall [30] that if $v$ is a continuous automorphism of $T$ (i.e. a linear map of $T), \bmod v=\operatorname{det} v$, and also that, since $N\left(T_{H}\right)$ is a semidirect product:

$$
\Delta_{N\left(T_{H}\right)}(a, r)=\Delta_{T}(a) \cdot \Delta_{Q_{H}}(r) \cdot \bmod r .
$$

Therefore $N\left(T_{H}\right)$ is always unimodular, since $\operatorname{det}(r)=1, Q_{H}$ is compact and $T$ is abelian.

\subsection{The Different Classes of Solutions}

a) Cases Listed in the Second Line of Table 1. Consider a discrete subgroup $Z^{3} \subset T$. Then $T / Z^{3}=\mathscr{T}_{3}$ the three dimensional torus, which is compact and has a finite $T$-invariant measure $m_{2}\left(\mathscr{T}_{3}\right)$.

The abelian discrete group $Z^{3}$ is unimodular, so $E / Z^{3}$ carries an invariant measure $m$. Topologically:

$$
E / Z_{3}=E / T \times T / Z_{3}=K \times \mathscr{T}_{3}
$$

so $E / Z_{3}$, product of topological spaces, is compact. Hence, the total measure $m$ is finite and equal to

$$
m\left(E / Z_{3}\right)=m_{1}(K) \cdot m_{2}\left(\mathscr{T}_{3}\right) .
$$

From Lemma 4 all closed subgroups $H$ of $E$ which contain a $Z^{3} C T$ are solutions of our problem. Those are the crystallographic groups in three dimensions $\left(T_{H}=Z_{3}\right)$, their generalization for "two dimensional" $\left(T_{H}=Z^{2}+R\right)$ and "one dimensional" $\left(T_{H}=Z+R^{2}\right)$ crystals in 3 dimensional space. This class of solutions contains also the subgroups of $E$ which are extensions of a closed subgroup ${ }^{10}$ of $K=S O$ (3) by the translation group $T$ (i.e. $T_{H}=R^{3} \approx T$ ).

To summarize, the solutions of case a) are all closed subgroups $H$ of $E$ whose $T_{H}=H \cap T$ is listed in (29'). The inclusion of subgroups $Z^{3} \subset H \subset E$ implies the existence of a canonical continuous surjective map $E / Z^{3} \stackrel{\varphi}{\longrightarrow} E / H$, so $E / H$, continuous image of a compact, is compact. Hence in case a) we verify, for $E$, Mostow's extended theorem.

10 The closed proper subgroups of $S O(3)$ are the finite groups: cyclic of order $n: C_{n}$ (rotations of $2 \pi \mathrm{k} / \mathrm{n}$ around an axis), dihedral $C_{n h}$ (add the rotations of $\pi$ around the perpendicular axes), and those related to the regular polyhedrons:tetrahedral, octahedral and icosahedral. The non finite ones are $S O(2)$ (all rotations around an axis), $\approx O(2)$ (add the rotations of $\pi$ around an axis $\perp$ ). 
b) Cases Listed in the First Line of Table 1, Except $T_{H}=\{0\}$. As we have seen at the end of 5.3, the $N\left(T_{H}\right)$ are unimodular, so $E / N\left(T_{H}\right)$ carries an invariant measure $m_{1}$. From (ii)

$$
E / N\left(T_{H}\right) \sim(E / T) /\left(N\left(T_{H}\right) / T\right)=K / Q_{H} .
$$

Thus, since $K / Q_{H}$ is compact, $m_{1}\left(E / N\left(T_{H}\right)\right)$ is finite. From our second strategical remark in 5.1, our problem for case b) is transformed into the problem for the $N\left(T_{H}\right)$, i.e. to find the closed unimodular subgroups $H \subset N\left(T_{H}\right)$ such that $H \cap T=T_{H}$ and the invariant measure $m^{\prime}\left(N\left(T_{H}\right) / H\right)$ is finite. From Mostow's theorem (iv), the last requirement is equivalent to impose $N\left(T_{H}\right) / H$ compact, so in case b) we also verify for $E$ Mostow's extended theorem.

Case b) now subdivides into

b 1) $Q_{H}$ finite. $\left(T_{H}=Z^{2}\right.$ or $\left.Z+R\right)$.

No solutions. Indeed, using (ii) we have the isomorphism

$$
N\left(T_{H}\right) / H \approx\left(N\left(T_{H}\right) / T_{H}\right) / K_{H},
$$

where $K_{H}=H / T_{H}$ is a subgroup of $Q_{H}$, thus is finite. We shall prove that $\left(N / T_{H}\right) / T_{H}$ is not compact, so that, using (i), $N\left(T_{H}\right) / H$ cannot be compact. The connected component $N_{0}\left(T_{H}\right)$ of $N\left(T_{H}\right)$ is $T$ and by (i) $N\left(T_{H}\right) / T_{H}$ is not compact because $T / T_{H}$ is not compact; indeed

$$
\begin{aligned}
& T_{H}=Z^{2} \Rightarrow T / T_{H}=R \times \mathscr{T}_{2} \\
& T_{H}=Z+R \Rightarrow T / T_{H}=R \times \mathscr{T}_{1}
\end{aligned}
$$

where $\mathscr{T}_{n}$ is the $n$-dimensional torus, which is compact; but $R$ is not.

b 2) $Q_{H} \approx O(2)\left(T_{H}=Z, R\right.$ or $\left.R^{2}\right)$.

We first solve the problem for $N_{0}\left(T_{H}\right)$, the connected component of $N\left(T_{H}\right)$; it is a subgroup of index 2 . It is the semi-direct product

$$
N_{0}\left(T_{H}\right)=R^{2} \square C_{2}
$$

where $C_{2}$ is the cylinder group, i.e. the abelian group generated by the rotations around the axis $\boldsymbol{u}$ and the translations along this axis. The universal covering $\bar{C}_{2}$ of $C_{2}$ is isomorphic to $R^{2}$ :

$$
\bar{C}_{2} \approx R^{2} \stackrel{p}{\longrightarrow} C_{2}
$$

and the fundamental group of $C_{2}$ is isomorphic to $Z \approx \operatorname{Ker} p$. 
The elements of $N\left(T_{H}\right)$ which do not belong to $N_{0}\left(T_{H}\right)$ contain moreover the rotation of $\pi$ around an axis $\perp \boldsymbol{u}$. Case b 2) now splits into the following three subcases:

$$
\text { b } 2 \propto) T_{H}=R^{2} \text {. }
$$

We look first for unimodular closed subgroups $H_{0}$ of $N_{0}\left(T_{H}\right)$ such that $N_{0}\left(T_{H}\right) / H_{0}$ is compact. Using (ii), $F=H_{0} / R^{2}$ is topologically isomorphic to a closed subgroup of $C_{2}$ such that $C_{2} / F$ is compact and $F \cap R_{0}=\{0\}$ where $R_{0}$ is the subgroup of translations along $\boldsymbol{u}$.

An equivalent characterization of the $F$ 's is

$$
\begin{gathered}
p^{-1}(F) \quad \text { is a closed subgroup of } \bar{C}_{2}, \\
\bar{C}_{2} / p^{-1}(F) \approx C_{2} / F \quad \text { is compact, } \\
p^{-1}(F) \cap p^{-1}\left(R_{0}\right)=\operatorname{Ker} p .
\end{gathered}
$$

Condition (37) requires (use [31]) that $p^{-1}(F) \approx Z^{p}+R^{q}$ with $0 \leqq p+q \leqq 2$, $p, q$ positive integers. Condition $\left(37^{\prime}\right)$ requires $p+q=2$, and conditions $\left(37^{\prime \prime}\right)$ require $q=0$. So $p^{-1}(F) \approx Z^{2}$ and the generators $f_{1}, f_{2}$ must satisfy:

$$
\left\{\begin{array}{l}
p\left(f_{1}\right) \quad \text { is a pure rotation of angle } \frac{2 \pi}{k}, k \text { positive integer, } \\
p\left(f_{2}\right)=\left(\lambda \boldsymbol{u}, r_{\theta}\right)
\end{array}\right.
$$

where $\lambda \boldsymbol{u}$ is a non-zero translation along $\boldsymbol{u}$ and $r_{\theta}$ is a rotation around $\boldsymbol{u}$ by an angle $\theta$ such that $\frac{\theta}{\pi}$ is irrational. Then $F \approx Z+Z_{k}$ where $Z_{k}=Z / k Z$.

The corresponding groups $H_{0}$ are the semi-direct products $R^{2} \square F$. They are solutions of our problem; the other solutions are obtained by adjoining to those $H_{0}$ the rotation of $\pi$ around one (and so all) axis $\perp \boldsymbol{u}$.

b $2 \beta) T_{H}=R$. No solutions:

Note first that the connected component of $N\left(T_{H}\right)$ has the structure $N_{0}\left(T_{H}\right) \approx R \times E_{2}$ direct product of $R$ and $E_{2}$, the connected euclidean group in two dimensions. Using (ii):

$$
N_{0}\left(T_{H}\right) / H \sim\left(N_{0}\left(T_{H}\right) / T_{H}\right) /\left(H / T_{H}\right)=E_{2} / K_{H}
$$

our problem is equivalent to finding the unimodular closed subgroup $K_{H}$ of $E_{2}$ such that $K_{H}$ contains no translation of $E_{2}$ and $E_{2} / K_{H}$ is compact. 
What has been written for the three dimensional Euclidean group $E(3)$ in 5.2 is valid for all $E(n)$ of dimension $n$. But the group $E(2)$ has properties which are not shared by the $E(n), n>2$; mainly

$-K \approx S O(2)$ is abelian,

$-\forall r \neq 1,1-r$ is an invertible endomorphism of $T$,

- every element of $E(2)$ which is not a translation is conjugated to a rotation; indeed (see $\left.\left(25^{\prime}\right),(26)\right)$.

$$
(a, r)=\left((1-r)^{-1} a, 1\right)(0, r)\left((1-r)^{-1} a, 1\right)^{-1} \in K_{2}\left((1-r)^{-1} a\right) .
$$

So if $X$ has only one generator $(a, r)$ it is a subgroup of $K_{2}\left((1-r)^{-1} a\right)$ and either it is finite so it is compact and $E / X$ is not compact (see (ii)) or it is infinite, so it is not closed in $K_{2}\left((1-r)^{-1} a\right)$ and hence in $E$. Hence the group $K_{H}$ that we are looking for must have at least two generators $(a, r),(b, s) r \neq 1 \neq s$ which are not in the same $K(t)$ i.e.

$$
(1-r)^{-1} a \neq(1-s)^{-1} b \Leftrightarrow(1-s) a-(1-r) b \neq 0
$$

but the commutator

$$
(a, r)(b, s)(a, r)^{-1}(b, s)^{-1}=((1-s) a-(1-r) b, 1)
$$

is a pure translation.

So there is no $K_{H}$, and therefore no $H$, solution of our problem when $T_{H}=R$.

b $2 \gamma) T_{H}=Z$. No solutions: for we can transform this case into a problem of case b $2 \beta$ ).

Indeed $H / T_{H}=K_{H}$ is a closed subgroup of $N\left(T_{H}\right) / T_{H}$ whose connected component is the direct product

$$
N_{0}\left(T_{H}\right) / T_{H} \approx S O(2) \times E_{2}
$$

because $R / Z=S O(2)$. Since $S O(2)$ is a compact subgroup as we noted in (ii), the canonical continuous map

$$
S O(2) \times E_{2} \stackrel{\varphi}{\rightarrow} E_{2}
$$

is closed. So $\varphi\left(K_{H}\right)$ is a closed subgroup of $E_{2}$.

Set $H \cap N_{0}\left(T_{H}\right)=H_{0}$; from the homeomorphisms

$$
N_{0}\left(T_{H}\right) / H_{0} \sim S O(2) \times E_{2} / K_{H_{0}}=E_{2} / \varphi\left(K_{H_{0}}\right),
$$

where $K_{H_{0}}=H_{0} / T_{H}$, we see that $\varphi\left(K_{H_{0}}\right)$ must be a closed subgroup of $E_{2}$ with compact homogeneous space. As we saw in b $2 \beta$ ), this is impossible. 
c) $T_{H}=0$. No Solution. The canonical map $\varphi$ defined in (24") has $\operatorname{ker} \varphi=T$, and $0=H \cap T$ implies that the groups $H$ and $\varphi(H)$ are isomorphic as abstract groups. The isomorphism is topological, $H \approx \varphi(H)$, iff $H$ is compact; then $\varphi(H)$ is closed. We have seen in 5.2 that compact subgroups of $E$ cannot be solution of our problem. Hence we have to look for $H$ such that $\varphi(H)$ is not closed in $K$. The closure $\overline{\varphi(H)}$, must be either $S O(2), O(2), S O(3)$ (see footnote of case a)). Before studying these three subcases, we divide the elements of $E$ into two classes:

$$
\left.\begin{array}{l}
\text { class } \mathrm{I}=\{(a, r), \boldsymbol{a} \cdot \boldsymbol{n}(r) \neq 0\} \\
\text { class II }=\{(a, r), \boldsymbol{a} \cdot \boldsymbol{n}(r)=0\}
\end{array}\right\}
$$

where $\boldsymbol{n}(r), \theta(r)$ denote the axis and the angle of the rotation $r, \boldsymbol{a}$ denotes the vector which represents the translation $a$, and $\boldsymbol{a} \cdot \boldsymbol{b}$ is the Euclidean scalar product.

If $(a, r)$ is in class I, we can decompose $\boldsymbol{a}$ into

$$
\boldsymbol{a}=\boldsymbol{a}_{\perp}+\boldsymbol{a}_{\|} \quad \text { with } \quad \boldsymbol{a}_{\perp} \cdot \boldsymbol{n}(r)=0, \boldsymbol{a}_{\|}=\alpha \boldsymbol{n}
$$

Then $\left(\right.$ see $\left.\left(25^{\prime}\right)\right)$

$$
(a, r)^{n}=\left(n a_{\|}+\left(1-r^{n}\right) a^{(r)}, r^{n}\right)
$$

where $a^{(r)}$ is a shorthand for

$$
a^{(r)}=\left(\left.(1-r)\right|_{\perp \boldsymbol{n}(r)}\right)^{-1} a_{\perp},
$$

i.e. the transform of $a_{\perp}$ by the inverse of the restriction of $(1-r)$ to the plane perpendicular to $\boldsymbol{n}(r)$. If $r$ is of finite order $n, r^{n}=1$ and $(a, r)^{n}=\left(n a_{\|}, 1\right)$. Since $H$ does not contain translations:

$$
(a, r) \text { in class } I \Rightarrow \theta(r) / 2 \pi \text { is irrational . }
$$

We now consider separately the three subcases.

$\mathrm{c} \alpha) \varphi(H)$ is Not Closed, $\overline{\varphi(H)} \approx S O(2)$.

Then all $r$ have the same $\boldsymbol{n}(r)$. If the elements of $H$ are all of class II, then $H \subset E(2)$ (the closed two dimensional Euclidean subgroups of $E$ for a plane perpendicular to $\boldsymbol{n}(r))$. We have seen that there are no solution to our problem in that case (see b $2 \beta)$ ). Hence $H$ has at least one element of class I. Consider first the case where $H$ is the infinite cyclic group $(\approx Z)$ with one generator of class I. It is closed: each point is at a distance strictly greater than $\left|\boldsymbol{a}_{\|}\right|$from the others; it is a closed subgroup (up to a linear transformation) of all groups solutions of case b $2 \alpha$ ). Let $H_{0}$ be the smallest one: $H_{0}=R^{2} \square Z$. Then $H_{0} / H \sim R^{2}$; since the $H_{0}$-invariant measure on $R^{2}$ (which is the Lebesgue measure) is not finite, due to our third strategical remark (end of 5.1), $H$ is not a solution of our problem. 
More generally this eliminates all closed $H^{\prime}$ of this case with one generator of class I and a finite number of elements of class II, since such $H^{\prime}$ are subgroups of a group $H$ solution of case b $2 \alpha$ ) with $H / H^{\prime} \sim R^{2}$. Groups $H$ with several independent generators of class I are eliminated because the commutator of two such generators is a translation (this argument has already been used at the end of case b $2 \beta)$ ).

c $\beta) \varphi(H)$ is Not Closed, $\overline{\varphi(H)} \approx O(2)$.

One must add to all subgroups $H$ studied in $\mathrm{c} \alpha$ ) at least a rotation of $\pi$ around an axis perpendicular to $\boldsymbol{n}(r)$ as defined in $\mathrm{c} \alpha$ ). The same arguments reject these extended groups as solutions of our problem.

$\mathrm{c} \gamma) \varphi(H)$ is Not Closed, but Dense in $K$.

In that case $H$ cannot be discrete: indeed one can prove (see e.g. [35], Lemma $3,2,7)$ that $H$ discrete implies $\varphi(\bar{H})$ abelian. So $H_{0}$, the connected component of the identity in $H$ cannot be reduced to a point. Since $\left.\varphi\right|_{H}$ is a continuous injective group isomorphism, $\varphi\left(H_{0}\right)$ is connected and does not reduce to a point and $\overline{\varphi\left(H_{0}\right)}$ is a connected closed subgroup of $K$ : i.e. either $S O(2)$ or $K$ itself. Consider a subgroup $A \approx S O(2) \subset \overline{\varphi\left(H_{0}\right)}$. Then $\varphi\left(H_{0}\right) \cap A$ is a connected subgroup of $A$; this is possible only if $\varphi\left(H_{0}\right) \cap A=A \approx S O(2)$. The density of $\varphi(H)$ implies the existence of at least $r \in \varphi(H)$ such that $r A r^{-1}=A^{\prime} \neq A$. Let $\boldsymbol{n}^{\prime}$ and $\boldsymbol{n}$ be the unit vectors of the rotation axis of $A^{\prime}$ and $A$. The set $C=A A^{\prime} A^{-1}$ contains all rotations of axis $\boldsymbol{n}^{\prime \prime}$ such that $\boldsymbol{n}^{\prime \prime} \cdot \boldsymbol{n}=\boldsymbol{n} \cdot \boldsymbol{n}^{\prime}=\cos \omega$. The set $C^{\prime}=A^{\prime} C A^{\prime-1}$ contains all rotations of axis $\boldsymbol{n}^{\prime \prime \prime}$ such that $\boldsymbol{n}^{\prime \prime \prime} \cdot \boldsymbol{n}^{\prime} \geqq \cos 2 \omega$. So $C^{\prime}$ is a neighbourhood of 1 in $K$ generating $K$. Therefore $\varphi(H)=K$, is closed, which is contradictory to our hypothesis.

\subsection{Conclusion}

We have established the list of all symmetry groups $G$ of transitive Euclidean states for the connected Euclidean group $E$. The extension to the full Euclidean group $E^{\prime}$ (including space symmetries) is trivial. $G$ is also a symmetry group of the physical system; it is then defined up to a conjugation in $E$. These symmetry groups are usually classified up to a conjugation in the general linear group. We have obtained five families of symmetry classes.

1. The crystallographic groups in 3 dimensions. They form 230 symmetry classes.

2. The groups generated by the group $R$ of translations along an axis $\boldsymbol{u}$ and the Euclidean transformations which induce a 2-dimensional crystallographic group (17 classes) on the plane $R^{2} \perp \boldsymbol{u}$; such transformations contain a discrete group $Z^{2}$ of translations in $R^{2}$, and eventually 
rotations around $\boldsymbol{u}$ (of angle multiple of $\frac{\pi}{3}$ or $\frac{\pi}{2}$ ), rotations of $\pi$ around an axis in $R^{2}$, symmetry through the $R^{2}$ plane or through a plane containing $\boldsymbol{u}$. This family contains a finite number of classes.

3. The groups generated by

i) the translations in a plane $R^{2}$,

ii) a translation $\lambda \boldsymbol{u}(\lambda \neq 0)$ along the direction $\boldsymbol{u} \perp R^{2}$,

iii) and zero, one, two or three of the following generators $\alpha, \beta, \sigma$.

$\alpha$ ) a rotation around $\boldsymbol{u}$ of angle rational to $\pi$,

$\beta$ ) a rotation of $\pi$ around an axis in the plane $R^{2}$,

$\gamma$ ) the symmetry through the plane $R^{2}$ or through a plane containing $\boldsymbol{u}$.

This family contains an infinity of symmetry classes and among them the 2 classes of 1-dimensional crystal group.

4. The semi-direct products $T \square K^{\prime}$ where $K^{\prime}$ is a closed subgroup of the orthogonal group in 3 dimensions (an infinity of classes).

5. The groups generated by

i) the translations in a plane $R^{2}$ (as in $3 \mathrm{i}$ ),

ii) a helicoidal transformation $(a, r)$ along the axis $\boldsymbol{u} \perp R^{2}$ with an angle $\theta(r)$ irrational to $\pi$, i.e. $a=\lambda \boldsymbol{u}, \lambda \neq 0, \boldsymbol{n}(r)=\boldsymbol{u}, \theta(r) / \pi$ irrational,

iii) as in 3 iii).

This family is generally forgotten in such a classification. It also contains an infinity of symmetry classes. When the angle $\theta$ of the generator in ii) becomes rational, the groups go from family 5 to family 3 . In each family, some symmetries only are realized in nature. Examples of symmetries in family 5 or 3 are the cholesteric liquids and the matter in a helimagnetic state, while ferromagnetism is an example of a broken Euclidean symmetry in family 4.

Acknowledgements. The authors would like to thank E. Calabi, A. Guichardet, R. V. Kadison, G. D. Mostow, D. Ruelle and S. Sakai for illuminating discussions. The first named author greatfully acknowledges the hospitality of II. Institut für Theoretische Physik der Universität Hamburg, Department of Mathematics, University of Pennsylvania, and Institute for Advanced Study, Princeton.

\section{References}

1. Kastler,D., Robinson,D.W.: Invariant states in statistical mechanics. Commun. math. Phys. 3, 151 (1966).

2. Robinson,D.W., Ruelle, D.: Extremal invariant states. Ann. Inst. Henri Poincaré, Sect. A 6, 299 (1967).

3. Doplicher, S., Kastler,D.: Ergodic states in a non commutative ergodic theory. Commun. math. Phys. 7, 1 (1968). 
4. _... Størmer, E.: Invariant states and asymptotic abelianness. J. Functional Anal. 3, 419 (1969).

5. Sakai, S.: On the central decomposition for positive functionals on $C^{*}$-algebras. Trans. Am. Math. Soc. 118, 406 (1965).

6. Guichardet, A., Kastler, D.: Décomposition des états quasi-invariants des $C^{*}$-algèbres. J. Math. Pures Appl. 49, 349 (1970).

6a. Ruelle, D.: Integral representation of states on a $C^{*}$-algebra. J. Functional Anal. 6, 116 (1970).

7. Dixmier, J.: Les $C^{*}$-algèbres et leurs représentations. Paris: Gauthier-Villars 1964.

8. Segal, I.E.: Irreducible représentations of operator algebras. Bull. Am. Math. Soc. 53, 73 (1947).

9. Gelfand,I.M., Neumark.M. A.: Normed rings with involution and their representations. Izsvest. Ser. Math. 12, 445 (1948).

10. Effros, E.: Convergence of closed subsets in a topological space. Proc. Am. Math. Soc. 16, 929 (1965).

10a. Feldman, J.: Borel sets of states and of representations. Michigan Math. J. 12, 363 (1965).

11. Kadison, R.V.: Transformation of states in operator theory and dynamics, topology. 3, Suppl. 2, 177 (1965).

12. Hille, E., Phillips, R.S.: Functional analysis and semi-groups. Am. Math. Soc. Coll. Publ. Providence R. I. (1957).

12a. Glicksberg de Leuw: J. Analyse Math., t. 15, 135-192(1965).

13. Segal, I.E.: A class of operator algebras which are determined by groups. Duke Math. J. 18, 221 (1951).

14. Doplicher, S., Kastler,D., Robinson, Derek W.: Covariance algebras in field theory and statistical mechanics. Commun. math. Phys. 3, 1 (1966).

15. Ruelle, D.: States of physical systems. Commun. math. Phys. 3, 133 (1966).

16. Doplicher, S., Kadison, R. V., Kastler, D., Robinson, Derek W.: Asymptotically abelian systems. Commun. math. Phys. 6, 101 (1967).

17. Størmer, E.: Large groups of automorphisms of $C^{*}$-algebras. Commun. math. Phys. 5, 1 (1967).

18. Bourbaki, N.: Livre III topologie générale, fascicule de résultats. Paris: Hermann 1964.

19. Neveu, J.: Bases mathématiques du calcul des probabilités. Paris: Masson 1964.

20. Mackey, G.W.: Induced representations of locally compact groups. Ann. Math. 55, 101 (1952).

21. - The theory of group representations. Mimeographed Chicago University Lecture Notes (1955).

22. - Unitary representations of group extensions I. Acta Math. 99, 265 (1958).

23. - Borel structures in groups and their duals. Trans. Am. Math. Soc. 85, 134 (1957).

24. Guichardet,A.: Utilisation des sous-groupes distingués dans l'étude des représentations unitaires des groupes localement compacts. Compos. Math. 17, 1 (1965).

25. Fell,J.M.G.: An extension of Mackey's method to representations of algebraic bundles I, II and III (to appear).

26. Zeller-Meier, G.: Produits croisés d'une $C^{*}$-algèbre par un groupe d'automorphismes. C. R. Acad. Sci. Paris, 263, 20 (1966).

27. - Produits croisés d'une $C^{*}$-algèbre par un groupe d'automorphismes. Preprint (1967).

28. Takesaki, M.: Covariant representation of $C^{*}$-algebras and their locally compact automorphisms groups (to appear).

29. Mostow, G. D.: Homogeneous spaces with finite invariant measure. Ann. Math. 75, 17 (1962). 
30. Bourbak1, N.: Livre VI intégration, ch. VII, Mesure de Haar. Paris: Hermann 1963.

31. See for instance $N$. Bourbaki III. Topologie générale, ch. VII, les groupes additıfs $R^{n}, \S 1, \mathrm{n}^{0} 2$ : "Sous-groupes fermés de $R^{n}$ ". Paris: Hermann 1967.

32. Bieberbach, L.: Examples of fundamental references for (i) (ii) (iii). Math. Ann. 70, 297 (1911).

33. See a Bourbaki: III Topologie générale, ch. 3, Groupes topologiques.

34. Hewitt, E., Ross, K.A.: Abstract harmonic analysis I. Berlin-Göttingen-Heidelberg: Springer 1963.

35. Wolf, J. A. : Spaces of constant curvature. New York: McGraw Hill 1966.

D. Kastler, M. Mebkhout

Centre de Physique Théorique

C.N.R.S.

31 Chemin J. Aiguier

F-13 Marseille 9, France

G. Loupias

Physique Mathématique

Université des Sciences et des Techniques

du Languedoc

Montpellier, France

L. Michel

Institut des Hautes Etudes Scientifique

91440 Bures-Sur-Yvette, France 